\title{
Vortex states of a disordered quantum Hall bilayer
}

\author{
P. R. Eastham, ${ }^{1}$ N. R. Cooper, ${ }^{2}$ and D. K. K. Lee ${ }^{1}$ \\ ${ }^{1}$ Blackett Laboratory, Imperial College London, London SW7 2AZ, United Kingdom \\ ${ }^{2}$ Cavendish Laboratory, University of Cambridge, Cambridge CB3 OHE, United Kingdom \\ (Received 30 March 2009; revised manuscript received 4 June 2009; published 1 July 2009)
}

\begin{abstract}
We present and solve a model for the vortex configuration of a disordered quantum Hall bilayer in the limit of strong and smooth disorder. We argue that there is a characteristic disorder strength below which vortices will be rare and above which they proliferate. We predict that this can be observed tuning the electron density in a given sample. The ground state in the strong-disorder regime can be understood as an emulsion of vortex-antivortex crystals. Its signatures include a suppression of the spatial decay of counterflow currents. We find an increase of at least an order of magnitude in the length scale for this decay compared to a clean system. This provides a possible explanation of the apparent absence of leakage of counterflow currents through interlayer tunneling, even in experiments performed deep in the coherent phase where enhanced interlayer tunneling is observed.
\end{abstract}

DOI: 10.1103/PhysRevB.80.045302

PACS number(s): 73.43.Nq, 73.43.Jn, 73.43.Lp

\section{INTRODUCTION}

There has been much recent progress in the search for quantum-condensed phases of quasiparticles in solids such as Bose-Einstein condensates of excitons, polaritons, and magnons. A very interesting example ${ }^{1-3}$ occurs for electron bilayers in the quantum Hall regime. When the two layers are close and have individual filling factors $\nu=1 / 2$, the Coulomb interactions produce a ground state in which electrons in one layer are correlated with holes in the other. The wave function of this state is that of a Bose-Einstein condensate of interlayer excitons, and it exhibits behaviors reminiscent of superfluidity and the Josephson effects: a small counterflow resistivity, ${ }^{4,5}$ which can be understood as excitonic superfluidity, and a zero-bias tunneling anomaly, ${ }^{6,7}$ which can be interpreted as a Josephson effect. However, the analogy is incomplete because neither the counterflow resistivity nor the width of the tunneling anomaly ${ }^{8}$ appears to vanish at finite temperatures.

Many theoretical works have suggested that these deviations from conventional superfluid behaviors are connected to the presence of vortices. In a quantum Hall system physical and topological charges are related so that random electric fields, created by the dopants, could induce vortices. The hypothesis that this leads to a disordered vortex state has been used ${ }^{9-11}$ to explain features such as the width of the tunneling anomaly and the region of negative differential conductance. More recently, Fertig and collaborators have developed a strong-disorder model, in which the dissipation reflects the dynamics of a vortex liquid. ${ }^{12,13}$ Despite these potential consequences, however, there have been few attempts to predict the vortex configuration in a bilayer. For weak layer-antisymmetric disorder the appropriate model is a gauge glass, ${ }^{12,14}$ suggesting vortex liquids, glasses, or conventional superfluid states are possibilities..$^{11,12,14-16}$ This is supported by exact diagonalization ${ }^{17}$ of small systems with white-noise disorder.

The aim of this paper is to predict the vortex configuration of a quantum Hall bilayer, for the case of strong long-range disorder, as is experimentally relevant for high-mobility modulation-doped samples. We argue that for a fixed disorder potential there is a characteristic value of the magnetic length, above which vortices proliferate. We find that this proliferation corresponds to the formation of an emulsion of vortex-antivortex crystals. Our theory should be testable since we estimate that the proliferation occurs in an experimentally accessible regime. Furthermore, we argue that the proliferation causes a dramatic suppression of the decay of counterflow currents. We find a length scale for this decay which is one to two orders of magnitude larger than the corresponding length scale in the clean system. This provides a possible explanation of a long-standing puzzle of the persistence of counterflow currents ${ }^{5}$ across an entire sample, in a regime where enhanced interlayer tunneling conductance is observed. Such behavior is quantitatively confirmed in recent experiments which show an area scaling for tunneling currents $^{18}$ up to the scale of $100 \mu \mathrm{m}$. More generally, our work suggests that the quantum Hall bilayer could be used to study a disordered form of the "supersolid" 19,20 that has previously attracted attention in superfluids, superconductors, and a clean bilayer model. ${ }^{21}$

The remainder of this paper is structured as follows. In Sec. II we develop a model for the vortex configuration of the bilayer and identify the parameters which control the vortex density. In Sec. III we present numerical results for the ground state of the model and compare these with a mean-field theory of an emulsion. In Sec. IV we analyze the decay of counterflow currents in the ground state, suggest some further consequences of the emulsion, and discuss the role of antisymmetric disorder. Finally, Sec. V summarizes our conclusions.

\section{MODEL}

We begin by developing a model for the vortex configuration, which we solve both numerically and in a mean-field approximation. Our starting point is the "coherencenetwork" picture ${ }^{12}$ in which the bilayer consists of compressible puddles of electron liquid, separated by channels of the incompressible counterflow superfluid (see Fig. 1). This is 


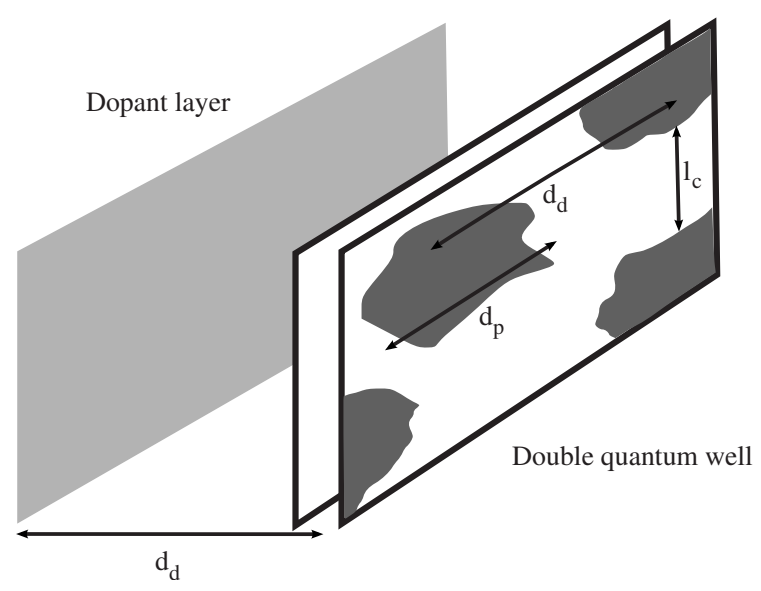

FIG. 1. Schematic of a disordered quantum Hall bilayer with compressible puddles of electron liquid (dark-shaded areas) of size $d_{p}$ surrounded by channels of incompressible excitonic superfluid of size $l_{c}$. For smooth disorder $d_{p} \sim l_{c} \sim d_{d}$ and the depicted length scales are larger than the magnetic length $l_{0}$. In the limit of very strong disorder, $l_{c}$ can become small and comparable to $l_{0}$.

appropriate for the strong smooth disorder produced by dopants, which destroys the superfluid over a significant fraction of the sample. ${ }^{22,23}$ We initially consider only layer-symmetric disorder since the distance to the dopants is much larger than the interlayer separation. We focus on the simplest case of a balanced bilayer, where the filling fraction in each layer is $\nu=1 / 2$, and initially also neglect the small interlayer tunneling.

This picture leads us to postulate the Hamiltonian

$$
H=\frac{1}{2} \sum_{i j}\left(Q_{i}-\bar{q}_{i}\right) E_{i j}\left(Q_{j}-\bar{q}_{j}\right)+\frac{1}{2} \sum_{i \neq j} v_{i} G_{i j} v_{j} .
$$

The first term is the electrostatic energy of an inhomogeneous charge distribution, written in terms of the charge $Q_{i}$ on the $i$ th compressible puddle, and the inverse capacitance matrix of the puddles $E_{i j}=C_{i j}^{-1}$. The potential due to the dopants is contained in the continuous-valued shifts $\bar{q}_{i}$ which would be the optimum charges on the puddles in classical electrostatics. This Coulomb term was not considered by previous work on the coherence network. We will see that it is the competition between Coulomb energy and superfluid stiffness that controls the proliferation of vortices in the system.

The second term in Eq. (1) models the energy of the channels. The condensate is characterized by a local phase $\theta(r)$, describing the interlayer phase coherence. Since this phase can wind by integer multiples of $2 \pi$ around each puddle, we associate vorticities $v_{i}$ with the puddles. The superfluid energy in the channels is $H_{\mathrm{sf}}=\int\left(\rho_{s} / 2\right)|\nabla \theta(\mathbf{r})|^{2} d^{2} \mathbf{r}$, with stiffness ${ }^{24,25} \rho_{s} \sim l_{0}^{-1}$. As usual, $H_{\text {sf }}$ leads to a vortex-vortex interaction $G_{i j} \sim-\log r_{i j}$ and a constraint $\Sigma_{i} v_{i}=0$.

The topological defects of the condensate are merons, ${ }^{25}$ which are vortices whose core corresponds to an unpaired electron in one layer. The meron charge is $q=(e / 2) \sigma v$, where $v$ is the vorticity and $\sigma= \pm 1$ denotes the layer index of the core. Because of this relationship the two terms in Eq. (1) are coupled and the charge disorder can drive vorticity in the channels. For those puddles with $|\bar{q} / e-[\bar{q} / e]|>1 / 4$ the electrostatics favors a half-electron charge, which is allowed if the vorticity around the puddle $v$ is odd. This costs a superfluid energy proportional to $v$. Therefore, a puddle will have $|v|=1$ if this incurs a superfluid-energy cost smaller than the electrostatic-energy gain.

These considerations allow us to identify the parameter controlling the vortex density in the percolating channels. For a wide range of parameters both the channel width $l_{c}$ and puddle size $d_{p}$ will be on the order of the distance to the dopant layers, $d_{d} \approx 200 \mathrm{~nm}^{26}$ (See Fig. 1 for illustration of these length scales.) The largest contribution to the electrostatic energy is the Coulomb interactions within each puddle. Thus we estimate the electrostatic-energy gain of a vortex as $E_{\text {cap }} \sim(1 / 2)(e / 2)^{2} / C$, where $C \sim d_{p} \sim d_{d}$ is the selfcapacitance of the puddle. We estimate the superfluid-energy cost as the prefactor of the vortex energy, which is generally $E_{s} \sim 2 \pi \rho_{s} \sim l_{0}^{-1}$. Thus the vortex density is controlled by the ratio

$$
\frac{E_{\text {cap }}}{E_{s}} \sim \frac{l_{0}}{d_{d}} .
$$

Since $d_{d}$ is fixed by the sample, we expect the vortex density to vary with the magnetic length.

In the limit of very strong disorder ${ }^{12} l_{c}$ becomes of the order of the magnetic length $l_{0} \approx 20 \mathrm{~nm}$ while $d_{p}$ remains of the order of $d_{d}$. The vortex energy in this regime is $E_{s}$ $\sim 2 \pi \rho_{s}\left(l_{c} / d_{d}\right)$, with the factor $l_{c} / d_{d}$ accounting for the fraction of the area occupied by the superfluid (up to numerical factors depending on the shapes of the puddles). Thus in the strong-disorder limit the vortex density becomes independent of $l_{0}, E_{\text {cap }} / E_{s} \sim 1$. We estimate this numerical parameter by modeling the puddles as disks of radius $d_{d} \approx 200 \mathrm{~nm}$ and taking $\rho_{s}$ from the mean-field theory ${ }^{25}$ at zero interlayer separation. This gives $E_{\mathrm{cap}} \approx E_{s} \approx 1 \mathrm{~K}$.

Since our estimates of $E_{\text {cap }}$ and $E_{s}$ in the strong-disorder limit are comparable, it may be possible to vary the density of vortices in experiments. Decreasing $l_{0}$ should take the system further from the (not unrealistic ${ }^{12,22,23}$ ) strongdisorder limit and so could lead to a reduction in the vortex density. More generally, reducing the vortex density requires a decrease in the capacitative energies, perhaps by placing gates on both sides of the sample as close as possible to the wells, or increasing the superfluid energy, perhaps in samples with smaller interlayer separation and larger tunneling.

To predict the vortex density and configuration of the bilayer, we now derive and solve a Hamiltonian for the vorticity. For simplicity we consider $E_{i j}=2 \delta_{i j} E_{\text {cap }}$. The offdiagonal terms will not qualitatively affect the results because the off-site Coulomb interactions have a much shorter range than the vortex interactions $G_{i j}$. The diagonal elements are approximately constant because they are controlled mainly by the characteristic puddle size. The main source of randomness is in the offset charges $\bar{q}_{i}$.

Taking $e / 2$ as our unit of charge, we write the total charge on each puddle as $Q_{i}=q_{i}^{M}+\sigma_{i} v_{i}$, where $\sigma= \pm 1, v=0, \pm 1$, and $q_{i}^{M}$ is the meron-free charge. In the ground state $q_{i}^{M}$ is the 

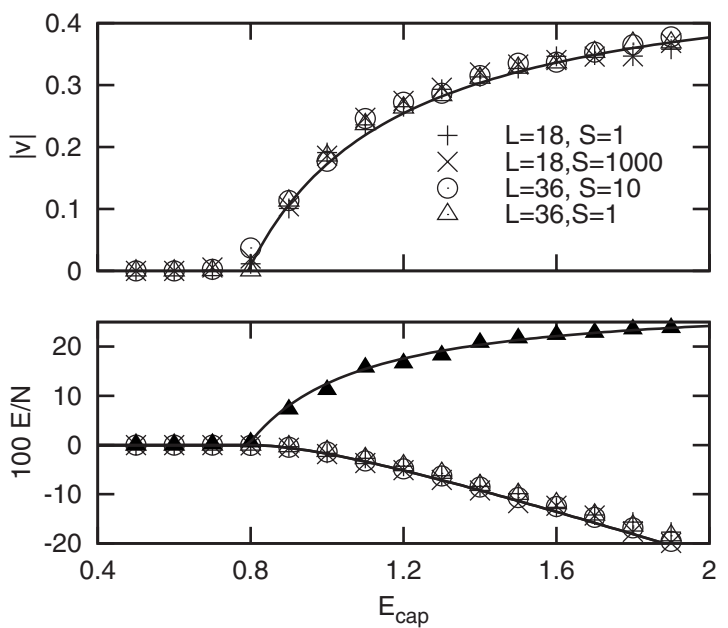

FIG. 2. Top panel: average ground-state vorticity $|v|$ as a function of disorder energy scale $E_{\text {cap }}$, obtained by simulated annealing on systems of linear size $L$, with $S$ Monte Carlo sweeps per temperature step (see text). Each point is an average over 25 disorder realizations. $\varepsilon_{i}$ is taken from a uniform distribution of width $2 E_{\text {cap }}$. Bottom panel: corresponding ground-state energies (crosses and hollow symbols) and interaction energy for $L=18, S=1$ (solid triangles). Solid curves show the corresponding results of the meanfield theory.

nearest even integer to $\bar{q}_{i}$. Thus the electrostatic energy of a vortex $v_{i}$ on site $i$ is

$$
E_{i}=E_{\text {cap }}\left[v_{i}^{2}+2 \sigma_{i} v_{i}\left(q_{i}^{M}-\bar{q}_{i}\right)\right]
$$

This is the only energy contribution which depends on the layer index of the core $\sigma_{i}$, and $E_{i}$ can be minimized by setting $\sigma_{i}=-\operatorname{sgn}\left[v_{i}\left(q_{i}^{M}-\bar{q}_{i}\right)\right]$. The distribution of $\bar{q}$ is broad on the scale of the charge quantization because the puddles contain many electrons so that $q_{i}^{M}-\bar{q}_{i}$ is a uniformly distributed random variable between \pm 1 . Thus, we see that the electrostatic energy takes the form $H=\Sigma_{i} \varepsilon_{i} v_{i}^{2}$, where $\varepsilon_{i}$ varies randomly from site to site, with distribution $P\left(\varepsilon_{i}\right)$. In the approximation that $E_{\text {cap }}$ is the same for all puddles, $\varepsilon_{i}$ is uniformly distributed between $\pm E_{\text {cap }}$. Note that in reality there will be some variation in $E_{\text {cap }}$ from puddle to puddle and the sharp edges in $P\left(\varepsilon_{i}\right)$ at $\pm E_{\text {cap }}$ will be smoothed out.

Combining the electrostatic and superfluid energies, we thus have an effective Hamiltonian for the vorticity

$$
H=\sum_{i} \varepsilon_{i} v_{i}^{2}+\frac{1}{2} \sum_{i \neq j} v_{i} G_{i j} v_{j} .
$$

We note that the random field is coupled to the presence of vortices, independent of their sign. This differs from gaugeglass models, where the random field couples directly to the vorticity.

\section{GROUND STATES}

Numerical results for the ground states of Eq. (4) are shown in Figs. 2 and 3. We adopt a lattice model (as in Ref. 12) where the channels are the edges of a square lattice of side $L$. We take the prefactor of the vortex energy $E_{s}$ to be
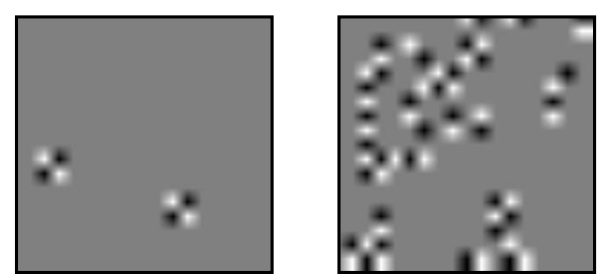

FIG. 3. Ground states for a typical realization of the disorder at strengths $E_{\text {cap }}=0.8$ (left) and 1.1 (right). Black/white are vorticities \pm 1 and gray is $0 . L=18$ and $S=1000$.

our unit of energy. Thus $G_{i j}=V\left(\mathbf{r}_{i j}\right)-V(0)$ is the lattice solution to $\nabla^{2} V(\mathbf{r})=-2 \pi \delta(0)$, with the singularity removed. ${ }^{19}$ Ground states were obtained by simulated annealing, with standard nearest-neighbor Monte Carlo moves. Each ground state is obtained by recording the lowest energy state obtained during an anneal, from a temperature of 0.5 to a temperature of 0.01 , in steps of 0.01 . At each temperature we perform $S$ sweeps of $4 L^{2}$ moves. As can be seen in Fig. 2, increasing $S$ by a factor of $10^{3}$ does not significantly change the results so we are obtaining good approximations to the ground states. The results in Fig. 2 are quenched averages of Monte Carlo data obtained for different disorder realizations.

From the top panel of Fig. 2 we see that the ground state is a uniform superfluid for small $E_{\text {cap }}$ while vortices proliferate above a threshold $E_{\text {cap }}^{0}$. The threshold behavior in $|v|$ as a function of $E_{\text {cap }}$ is sharp due to the discontinuity in the onsite energy distribution $P\left(\varepsilon_{i}\right)$ and would in reality be rounded due to the variations in $E_{\text {cap }}$ between puddles.

Figure 3 shows ground states obtained for a typical disorder realization at two different strengths. These results show that the vortex ground states are not completely disordered and are strongly suggestive of an emulsion of vortexantivortex crystals. This structure appears because the field in Eq. (4) does not dictate the sign of the vorticity. On the square lattice there is a minimum in the interaction $G_{\mathbf{q}}$ $=\pi / 8=\mu_{c}$ at wave vector $\mathbf{q}=(\pi, \pi)$ so for a uniform field $\varepsilon_{i}<-\mu_{c}$ the ground state is a vortex-antivortex crystal. ${ }^{19}$ Whereas a random-field coupling to $v_{i}$ (as in a gauge-glass model $^{12,14}$ ) competes with this ordering, the random-field coupling to $v_{i}^{2}$ does not. It can therefore straightforwardly induce regions of the crystalline phase.

The vortex density in Fig. 2 appears to be consistent with a mean-field theory of an emulsion. To develop such a theory, let us consider a mixture of two phases occupying fractions $\bar{x}$ and $(1-\bar{x})$ of the system, with energy densities $\mu_{c}$ and 0 , respectively. Without a random field the mean-field energy of such a mixture is ${ }^{27}$

$$
E=\mu_{c} \bar{x}+\kappa \bar{x}(1-\bar{x})
$$

where $\kappa$ is an interaction parameter, which corrects for the use of bulk energy densities in the first term. It will be the only fitting parameter in the theory.

To incorporate the random $\varepsilon_{i}$, we interpret Eq. (5) as a mean-field approximation for the microscopic effective Hamiltonian 


$$
H=\sum_{i} h x_{i}+\sum_{\langle i j\rangle} 2 J_{i j} x_{i} x_{j}
$$

where $x_{i}=0$ denotes a site in the vortex-free phase and $x_{i}$ $=1$ denotes one in the vortex-crystal phase. The mean-field approximation is obtained by writing $x_{i}=\bar{x}+\left(x_{i}-\bar{x}\right)$ and discarding terms quadratic in the fluctuations. Demanding that the resulting energy agree with Eq. (5) allows us to relate $h$, and the average $J_{i j}$ to $\mu_{c}$ and $\kappa$. We then incorporate the random-field term from Eq. (4), $\Sigma_{i} \varepsilon_{i} x_{i}$, to obtain

$$
H_{\mathrm{mf}}=\bar{x}^{2} \kappa N+\sum_{i}\left(\mu_{c}+\varepsilon_{i}+\kappa-2 \kappa \bar{x}\right) x_{i} .
$$

The mean-field equation is $\bar{x}=\left\langle x_{i}\right\rangle$, where \langle\rangle denotes an average in the ground state of Eq. (7). For the uniform distribution of width $2 E_{\text {cap }}$ for $\varepsilon_{i}$, we find that, when $\mu_{c}+\kappa$ $<E_{\text {cap }}$,

$$
\bar{x}=\frac{1}{2}\left(1-\frac{\mu_{c}}{E_{\text {cap }}-\kappa}\right),
$$

and $\bar{x}=0$ otherwise. We can also compute the energy,

$$
\frac{1}{N}\left\langle H_{\mathrm{mf}}\right\rangle=\bar{x}^{2} \kappa+\int_{\mu_{c}+\kappa-E_{\mathrm{cap}}-2 \kappa \bar{x}}^{0} \frac{E}{2 E_{\mathrm{cap}}} d E .
$$

The solid lines in Fig. 2 show the mean-field predictions of Eqs. (5), (8), and (9), with $\kappa=0.4$ chosen to give the threshold $E_{\text {cap }}$ obtained numerically. As can be seen, this theory, with a single fitting parameter, gives a good account of the numerical results. Thus the ground-state vorticity of Eq. (4) can indeed be understood in terms of the formation of an emulsion of vortex crystals.

\section{DISCUSSION}

The presence of the vortex-crystal emulsion would affect counterflow and tunneling experiments. Let us consider, in particular, the decay of a dc counterflow current due to tunneling. Without the vortices, the superfluid phase $\theta$ is obtained by minimizing the energy

$$
H=\int\left[\frac{\rho_{s}}{2}|\nabla \theta|^{2}-\Delta n \cos (\theta)\right] d^{2} \mathbf{r},
$$

where $\Delta$ is the tunneling strength and $n=1 /\left(2 \pi l_{0}^{2}\right)$ is the electron density. A small static perturbation to the solution $\theta=0$, such as a small counterflow current injected at one edge, decays on the scale set by the Josephson length,

$$
\lambda_{J} \sim l_{0} \sqrt{\frac{\rho_{s}}{\Delta}},
$$

estimated $^{9}$ as $\sim 5 \mu \mathrm{m}$. This means we should not expect counterflow currents to persist over more than a few microns due to leakage by interlayer tunneling (in other words, by the recombination of the interlayer excitons). This appears inconsistent with the experimental observation ${ }^{18}$ of an area scaling for the tunneling anomaly, up to length scales of $100 \mu \mathrm{m}$.

With pinned vortices, we should instead consider the energy associated with the vorticity-free part of the supercurrents. ${ }^{9}$ If we write the phase field of the vortices as $\theta_{0}$, we can separate out the vorticity-free phase field $\phi=\theta$ $-\theta_{0}$. For a fixed vortex field $\theta_{0}$, the ground state of the system is determined by a random-field $X Y$ model for the vorticity-free part of the system:

$$
H_{\phi}=\int\left[\frac{\rho_{s}}{2}|\nabla \phi|^{2}-\Delta n \cos \left(\phi+\theta_{0}\right)\right] d^{2} \mathbf{r} .
$$

This may be treated using standard techniques. ${ }^{28,29}$ In the emulsion, the pinning phase $\theta_{0}$ is disordered. We see that, in the limit of a vanishing correlation length for $\theta_{0}$, the tunneling field has no effect because it averages to zero. In our case, the vortex phase field has a correlation length $\xi \sim d_{d}$ $\ll \lambda_{J}$, corresponding to the weak-disorder regime of the random-field model. In this regime, the ground state $\phi$ consists of domains of linear size $L_{\text {dom }}$, aligned with the average random field across the domain. The typical tunneling energy in the random field is given by the sum of random energies in the range $\pm \Delta n \xi^{2}$ for $\left(L_{\mathrm{dom}} / \xi\right)^{2}$ correlation areas. This gives a typical energy of $\Delta\left(\xi / l_{0}\right)^{2} \sqrt{\left(L_{\mathrm{dom}} / \xi\right)^{2}}$. The cost in phase stiffness in the domain is on the order of $\rho_{s}\left(L_{\mathrm{dom}}\right)^{0}$ in two dimensions. Balancing these two energies, we find the domain size

$$
L_{\mathrm{dom}} \sim \lambda_{J}\left(\frac{\lambda_{J}}{\xi}\right) .
$$

We estimate that the Josephson length $\lambda_{J} \sim 5 \mu \mathrm{m}$ while the correlation length $\xi \sim 100 \mathrm{~nm}$. Therefore, this domain size $L_{\text {dom }}$ is an emergent length scale associated with the emulsion that could be one to two orders of magnitude larger than $\lambda_{J}$ in the clean system. Moreover, we see that static perturbations to this disordered ground state $(\phi \rightarrow \phi+\delta \phi)$, such as an injected counterflow current, decay over the length scale $L_{\text {dom. }}$. Allowing for the considerable uncertainty in $\lambda_{J}$, this decay length $(\sim 0.3 \mathrm{~mm})$ predicted by our model is consistent with the apparent experimental bound ${ }^{18}$ $(\gg 0.1 \mathrm{~mm})$. This should be contrasted with the vortex-free state which, as mentioned above, gives $\lambda_{J} \sim 5 \mu \mathrm{m}$ as the decay length.

The vortices in the emulsion will not be completely pinned and hence their presence will affect the counterflow superfluidity. Even if the vortices remain pinned to the puddles, they can move a distance $d_{p}$ across them, leading to a reduction in the stiffness. ${ }^{20}$ Thermally activated hopping of vortices between the puddles may lead to dissipation, as in previous work on the coherence network, ${ }^{12,13}$ so that the emulsion may formally be a vortex liquid at finite temperatures. However, the distribution of $\varepsilon_{i}$ in our model suggests a distribution of activation energies, in contrast to previous work.

Direct tests of our theory may be possible in imaging experiments. ${ }^{30}$ For example, our model predicts that charging lines corresponding to half-electron charges are common only when $E_{c} \gtrsim E_{s}$. More generally, the identity of physical and topological charges implies that the vortex configuration affects the charging spectra.

Finally, let us revisit the role of layer-antisymmetric disorder. It will give additional terms in Eq. (3) which are proportional to $\sigma_{i}$, leading to terms linear in $v_{i}$ in the Coulomb 
gas [Eq. (4)]. Provided the compressible puddles are effective at screening the antisymmetric disorder, the energy of the charge imbalance $\sigma_{i}$ will be approximately $e^{2} / C_{M}$, where $C_{M} \sim d_{p}^{2} / l_{0}$ is the mutual capacitance of two puddles in opposite layers. This energy is a factor of $l_{0} / d_{p} \ll 1$ smaller than $E_{\text {cap }}$ and the terms in $v_{i}$ are small compared with those in $v_{i}^{2}$. Thus while layer-antisymmetric disorder could affect correlations on very long scales, it will not affect the physical consequences described above, which are controlled by the scale $d_{p}$.

\section{CONCLUSIONS}

In conclusion, we have developed a model of a disordered quantum Hall bilayer, in the experimentally relevant limit of strong smooth disorder. We have argued that the ground state of this model can be understood as an emulsion of vortexantivortex crystals. Our theory suggests that the density of the emulsion could vary significantly with magnetic length, and between samples, allowing its effects to be isolated experimentally. An important physical consequence of the presence of such an emulsion (or other disordered vortex state) is a suppression of the decay of counterflow currents, potentially explaining the area scaling of the tunneling anomaly. ${ }^{18}$

\section{ACKNOWLEDGMENTS}

This work was supported by EPSRC-GB under Grant No. EP/C546814/01. We thank A. Stern for a critical reading of the manuscript and P. Littlewood for discussions.
${ }^{1}$ J. P. Eisenstein and A. H. MacDonald, Nature (London) 432, 691 (2004).

${ }^{2}$ S. Q. Murphy, J. P. Eisenstein, G. S. Boebinger, L. N. Pfeiffer, and K. W. West, Phys. Rev. Lett. 72, 728 (1994).

${ }^{3}$ T. S. Lay, Y. W. Suen, H. C. Manoharan, X. Ying, M. B. Santos, and M. Shayegan, Phys. Rev. B 50, 17725 (1994).

${ }^{4}$ E. Tutuc, M. Shayegan, and D. A. Huse, Phys. Rev. Lett. 93, 036802 (2004).

${ }^{5}$ M. Kellogg, J. P. Eisenstein, L. N. Pfeiffer, and K. W. West, Phys. Rev. Lett. 93, 036801 (2004).

${ }^{6}$ I. B. Spielman, J. P. Eisenstein, L. N. Pfeiffer, and K. W. West, Phys. Rev. Lett. 84, 5808 (2000).

${ }^{7}$ L. Tiemann, W. Dietsche, M. Hauser, and K. von Klitzing, New J. Phys. 10, 045018 (2008).

${ }^{8}$ J. P. Eisenstein, Solid State Commun. 127, 123 (2003).

${ }^{9}$ M. M. Fogler and F. Wilczek, Phys. Rev. Lett. 86, 1833 (2001).

${ }^{10}$ A. Stern, S. M. Girvin, A. H. MacDonald, and N. Ma, Phys. Rev. Lett. 86, 1829 (2001).

${ }^{11}$ L. Balents and L. Radzihovsky, Phys. Rev. Lett. 86, 1825 (2001).

${ }^{12}$ H. A. Fertig and G. Murthy, Phys. Rev. Lett. 95, 156802 (2005).

${ }^{13}$ B. Roostaei, K. J. Mullen, H. A. Fertig, and S. H. Simon, Phys. Rev. Lett. 101, 046804 (2008).

${ }^{14}$ A. Stern, S. Das Sarma, M. P. A. Fisher, and S. M. Girvin, Phys. Rev. Lett. 84, 139 (2000).

${ }^{15}$ M. P. A. Fisher, Phys. Rev. Lett. 62, 1415 (1989).
${ }^{16}$ R. A. Hyman, M. Wallin, M. P. A. Fisher, S. M. Girvin, and A. P. Young, Phys. Rev. B 51, 15304 (1995).

${ }^{17}$ D. N. Sheng, L. Balents, and Z. Wang, Phys. Rev. Lett. 91, 116802 (2003).

${ }^{18}$ A. D. K. Finck, A. R. Champagne, J. P. Eisenstein, L. N. Pfeiffer, and K. W. West, Phys. Rev. B 78, 075302 (2008).

${ }^{19}$ J. R. Lee and S. Teitel, Phys. Rev. B 46, 3247 (1992).

${ }^{20}$ M. Gabay and A. Kapitulnik, Phys. Rev. Lett. 71, 2138 (1993); Shou-Cheng Zhang, ibid. 71, 2142 (1993).

${ }^{21}$ I. Tupitsyn, M. Wallin, and A. Rosengren, Phys. Rev. B 53, R7614 (1996).

${ }^{22}$ A. L. Efros, Solid State Commun. 65, 1281 (1988).

${ }^{23}$ N. R. Cooper and J. T. Chalker, Phys. Rev. B 48, 4530 (1993).

${ }^{24}$ X. G. Wen and A. Zee, Phys. Rev. Lett. 69, 1811 (1992).

${ }^{25}$ K. Moon, H. Mori, K. Yang, S. M. Girvin, A. H. MacDonald, L. Zheng, D. Yoshioka, and S.-C. Zhang, Phys. Rev. B 51, 5138 (1995).

${ }^{26}$ J. P. Eisenstein (private communication).

${ }^{27}$ C. J. Adkins, Equilibrium Thermodynamics, 3rd ed. (Cambridge University Press, Cambridge, London., 1983), p. 231; B. Spivak, Phys. Rev. B 67, 125205 (2003).

${ }^{28}$ Y. Imry and S.-K. Ma, Phys. Rev. Lett. 35, 1399 (1975).

${ }^{29}$ H. Fukuyama and P. A. Lee, Phys. Rev. B 17, 535 (1978).

${ }^{30}$ J. Martin, S. Ilani, B. Verdene, J. Smet, V. Umansky, D. Mahalu, D. Schuh, G. Abstreiter, and A. Yacoby, Science 305, 980 (2004). 